June 2007

\title{
Deformations of Bundles and the Standard Model
}

\author{
Björn Andreas 12 and Gottfried Curio ${ }^{13}$ \\ ${ }^{2}$ Institut für Mathematik, Freie Universität Berlin \\ Arnimallee 14, 14195 Berlin, Germany \\ ${ }^{3}$ Institut für Mathematik, Humboldt-Universität zu Berlin \\ Rudower Chaussee 25, 12489 Berlin, Germany
}

\begin{abstract}
We modify a recently proposed heterotic model hep-th/0703210, giving three netgenerations of standard model fermions, to get rid of an additional $U(1)$ factor in the gauge group. The method employs a stable $S U(5)$ bundle on a Calabi-Yau threefold admitting a free involution. The bundle has to be built as a deformation of the direct sum of a stable $S U(4)$ bundle and the trivial line bundle.
\end{abstract}

\footnotetext{
${ }^{1}$ supported by DFG-SFB 647 (G. C. is also supported by the MPI für Physik, München)
} 
In this note, which constitutes an addendum to [1], we propose a method to construct a model of the $E_{8} \times E_{8}$ heterotic string giving in four dimensions the gauge group and chiral matter content of the standard model. For this we embed an $S U(5)$ bundle in the first $E_{8}$ leading to a GUT gauge group $S U(5)$, which is afterwards broken by a Wilson line to the standard model gauge group. The non-simply connected CalabiYau threefold is obtained by modding a simply connected cover Calabi-Yau space $X$ by a free involution. Therefore we search on $X$ for an invariant $S U(5)$ bundle of net-generation number \pm 6 (for other constructions along these lines cf. [2], [3], [4] ).

Models of this kind were recently constructed in [1] (cf. also [5]) but had an additional $U(1)$ in the unbroken gauge group due to the specific form of the bundle

$$
V_{5}=V_{4} \otimes \mathcal{O}_{X}\left(-\pi^{*} \beta\right) \oplus \mathcal{O}_{X}\left(4 \pi^{*} \beta\right)
$$

This is a polystable bundle and has structure group $S U(4) \times U(1)_{A}$ (on the Lie algebra level) of $V_{5}$ and therefore $S U(5) \times U(1)_{A}$ as unbroken gauge group.

All the conditions, stability of the bundle, invariance under the involution, solution of the anomaly cancellation equation by having an effective five-brane class and finally the phenomenologically net-generation number were therefore essentially solved already on the level of $V_{4}$. The bundle $V_{4}$ alone would give an unbroken gauge group $S O(10)$, which cannot be broken to the standard model gauge group by just turning on a $\mathbf{Z}_{2}$ Wilson line corresponding to $\pi_{1}\left(X / \mathbf{Z}_{2}\right)$. Therefore in [1] $V_{4}$ had to be enhanced to an $S U(5)$ bundle by adding a line bundle (the combined conditions of stability and fivebrane effectivity make a non-trivial extension for $V_{5}$ problematical as explored in [1]). Then the structure (0.1) caused the additional $U(1)_{A}$ in the gauge group.

So in a $(4+1)$-decomposition of the rank 5 structure group one has $\left(\begin{array}{l|l}a & 0 \\ \hline 0 & d\end{array}\right) \in$ $S U(5)$ where the $U(1)_{A}$ is embedded as $\left(\begin{array}{lll|ll}1 & & & & \\ & 1 & & & \\ & & 1 & & \\ & & & 1 & \\ & & & -4\end{array}\right)$. Our goal is to turn on the off-diagonal block elements to get a full, irreducible $S U(5)$, i.e. $\left(\begin{array}{l|l}a & * \\ \hline * & d\end{array}\right)$. The possibility to do this will be measured (in first order) be two Ext $t^{1}$-groups, corresponding to each of the off-diagonal blocks, respectively. The process of turning on these off-diogonal terms means that the bundle $V^{\prime}=V_{4} \oplus \mathcal{O}_{X}$ (for simplicity we set $\beta=0$ ) is deformed to a more generic bundle $V$; conversely $V^{\prime}$ occurs as a specialization or 
degeneration $V \rightarrow V^{\prime}$ where the off-diagonal terms again go to zero and one gets the reducible object. More precisely, we say a vector bundle $V^{\prime}$ deforms to a stable bundle $V$ if there is a connected curve $C$ and a vector bundle $\mathcal{V}$ over $C \times X$ such that $V^{\prime} \cong \mathcal{V}_{\{0\} \times X}$ for some point $0 \in C$ and $\mathcal{V}_{\{t\} \times X} \cong V$ for some other point $t \in C$

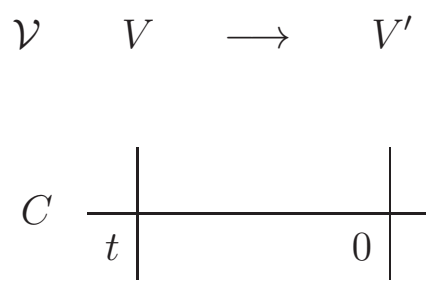

This process will thereby cause the following changes in the structure group $G$ of the bundles and the unbroken gauge group $H$ of the four-dimensional low-energy observer who sees the commutator in $E_{8}$ of $G$

\begin{tabular}{c|ccc} 
& $V$ & $\longrightarrow$ & $V^{\prime}$ \\
\hline$G$ & $S U(5)$ & & $S U(4) \times U(1)_{A}$ \\
$H$ & $S U(5)$ & & $S U(5) \times U(1)_{A}$
\end{tabular}

For a generic choice of parameters with $\alpha \beta \neq 0$ (where $\alpha \in H^{11}(B)$ is a further twist class inherent in the construction, cf. [1] and below) one would find that the $U(1)_{A}$, which occurs in the structure group and in the gauge group, is anomalous and thereby gets massive by the Green-Schwarz mechanism. However the group theory of the ad $E_{8}$ decomposition relevant here tells us that to secure the absence of exotic matter multiplets one has just to impose the condition $\alpha \beta=0$ (cf. [1]). Thereby the $U(1)_{A}$ remains non-anomalous and remains in the light spectrum. It is this problem for which the present paper shows a way out by embedding the reducible bundle in a family of proper irreducible $S U(5)$ bundles where the $U(1)_{A}$ is therefore again massive on the compactification scale (as all other elements of $E_{8}$ which are broken for the fourdimensional low-energy observer by the specific gauge background turned on on $X$ ); for the specialization $* \longrightarrow 0$, where the off-diagonal elements are turned off again, one would then get a restauration of four-dimensional gauge symmetry as this corresponds to $m_{U(1)_{A}} \longrightarrow 0$, i.e., at this special point on the boundary of the bundle moduli space the $U(1)_{A}$ returns into the light spectrum.

So, to get rid of this additional $U(1)_{A}$ factor we will construct a stable holomorphic $S U(5)$ bundle $V_{5}$ by deforming the complex structure of the given polystable $S U(5)$ 
bundle $V^{\prime}=V_{4} \oplus \mathcal{O}_{X}$ (as said, for simplicity we work from now on with $\beta=0$ ). $V_{4}$ is a stable $S U(4)$ bundle, and $\mathcal{O}_{X}$ is the trivial one-dimensional line bundle; therefore $V^{\prime}$ is a polystable bundle and solves the Donaldson-Uhlenbeck-Yau (DUY) equations. If such a deformation to a stable holomorphic bundle exist, the theorems of [6], [7] guarantee that $V_{5}$ is a solution of the DUY equations, i.e., the equations of motion of the heterotic string.

In [10] (Corollary B.3) it has been shown that the direct sum of two stable vector bundles (say $V, W$ ) of the same slope $\mu(V)=\int c_{1}(V) J^{2} / r k(V)$ deforms to a stable vector bundle if the sum has unobstructed deformations and both spaces $H^{1}(X, \operatorname{Hom}(V, W))$ and $H^{1}(X, H o m(W, V))$ do not vanish. Applied to our case we therefore have to show that $H^{1}\left(X, \operatorname{Hom}\left(V_{4}, \mathcal{O}_{X}\right)\right)$ and $H^{1}\left(X, \operatorname{Hom}\left(\mathcal{O}_{X}, V_{4}\right)\right)$ do not vanish and that $V_{4} \oplus \mathcal{O}_{X}$ has unobstructed deformations.

$H^{1}\left(X, \operatorname{End}\left(V^{\prime}\right)\right)$ is the space of all first-order deformations of $V^{\prime}$. The obstruction to extending a first order deformation to second (or higher) order lives in $H^{2}\left(X, \operatorname{End}_{0}\left(V^{\prime}\right)\right)$. Thus if $H^{2}\left(X, \operatorname{End}_{0}\left(V^{\prime}\right)\right)=0$ we can always lift to higher order, i.e., the deformations would be unobstructed. For instance, the tangent bundle $T X$ of a $K 3$ surface has unobstructed deformations since $H^{2}\left(X, \operatorname{End}_{0}(T X)\right) \cong$ $H^{0}\left(X, \operatorname{End}_{0}(T X)\right)^{*}=0$. As we are on a Calabi-Yau threefold, Serre duality shows that the dimensions of $H^{1}\left(X, \operatorname{End}\left(V^{\prime}\right)\right)$ and $H^{2}\left(X, \operatorname{End}\left(V^{\prime}\right)\right)$ are equal, so there are as many obstructions as deformations.

The vanishing of $H^{2}\left(X, \operatorname{End}\left(V^{\prime}\right)\right)$ is, however, only a sufficient condition for the existence of a global (in contrast to first-order) deformation. So in principle it is still possible to have global deformations although the obstruction space is non-vanishing. That this hope is not in vain is born out by the example of $X$ being the quintic in $\mathbf{P}^{4}$ and $V^{\prime}=T X \oplus \mathcal{O}_{X}$ (this example was considered first in [12], [13]), where nevertheless knows that a global deformation exists [10], [11].

The tangent space $H^{1}\left(X, \operatorname{End}\left(V^{\prime}\right)\right)$ to the deformations decomposes as follows (using the fact that $\left.H^{1}\left(X, \mathcal{O}_{X}\right)=0\right)$

$$
H^{1}\left(X, \operatorname{End}\left(V^{\prime}\right)\right) \cong H^{1}\left(X, \operatorname{End}\left(V_{4}\right)\right) \oplus H^{1}\left(X, H o m\left(V_{4}, \mathcal{O}_{X}\right)\right) \oplus H^{1}\left(X, H o m\left(\mathcal{O}_{X}, V_{4}\right)\right)
$$

where the last two terms $\operatorname{Ext}^{1}\left(V_{4}, \mathcal{O}_{X}\right)=H^{1}\left(X, V_{4}^{*}\right)$ and $\operatorname{Ext}^{1}\left(\mathcal{O}_{X}, V_{4}\right)=H^{1}\left(X, V_{4}\right)$ 
parametrize non-trivial extensions

$$
\begin{aligned}
& 0 \rightarrow \mathcal{O}_{X} \rightarrow W \rightarrow V_{4} \rightarrow 0 \\
& 0 \rightarrow V_{4} \rightarrow W^{\prime} \rightarrow \mathcal{O}_{X} \rightarrow 0
\end{aligned}
$$

We note first that the index theorem gives (by stability of $V_{4}$ and $\mu\left(V_{4}\right)=0$ we have $H^{i}\left(X, V_{4}\right)=0$ for $i=0,3$; note that also $V^{*}$ is stable and has $\left.\mu\left(V^{*}\right)=0\right)$

$$
\operatorname{dim} H^{1}\left(X, V_{4}\right)-\operatorname{dim} H^{1}\left(X, V_{4}^{*}\right)=-\frac{1}{2} c_{3}\left(V_{4}\right)
$$

which for the physical relevant $V_{4}$ is non-zero, so at least one of the two off-diagonal spaces is already non-vanishing. To actually prove that $H^{1}\left(X, V_{4}\right)$ and $H^{1}\left(X, V_{4}^{*}\right)$ are both non-vanishing we recall first the explicit construction of $V_{4}$ form [1]. Note that whereas in [1] we considered the case $x>0, \beta \neq 0$, we consider here the case $x<0$ (this simplifies some arguments below) and $\beta=0$.

As in [1] the rank four vector bundle $V_{4}$ will be constructed as an extension

$$
0 \rightarrow \pi^{*} E_{1} \otimes \mathcal{O}_{X}(-D) \rightarrow V_{4} \rightarrow \pi^{*} E_{2} \otimes \mathcal{O}_{X}(D) \rightarrow 0
$$

where $E_{i}$ are stable bundles on $B$ and $D=x \Sigma+\pi^{*} \alpha$ is a divisor in $X$ (here $X$ is a Calabi-Yau threefold elliptically fibered with two sections $\sigma_{i}$ over $B=\mathbf{P}^{1} \times \mathbf{P}^{1}$, cf. [1]; furthermore $\Sigma=\sigma_{1}+\sigma_{2}$ and $F$ will denote the fiber). The argument for stability of $V_{4}$ runs exactly parallel to the one given in [1]. To prove stability of $V_{4}$ we first note that given zero slope stable vector bundles $E_{i}$ on $B$, one can prove that the pullback bundles $\pi^{*} E_{i}$ are stable on $X$ [8], [9] for a suitable Kähler class $J$. Now for the zero slope bundle $V_{4}$ constructed as an extension (with $\pi^{*} E_{i}$ stable) we have two immediate conditions which are necessary for stability: first that $\mu\left(\pi^{*} E_{1} \otimes \mathcal{O}_{X}(-D)\right)<0$ and second that $\pi^{*} E_{2} \otimes \mathcal{O}_{X}(D)$ of $\mu\left(\pi^{*} E_{2} \otimes \mathcal{O}_{X}(D)\right)>0$ is not a subbundle of $V_{4}$, i.e., the extension is non-split. The first condition reduces to

$$
D J^{2}=2 x(h-z)^{2} c_{1}^{2}+2 z(2 h-z) \alpha c_{1}>0
$$

(where $\left.c_{i}:=\pi^{*} c_{i}(B)\right)$ this implies in our case $x<0$ the condition

$$
\alpha c_{1}>0
$$

The non-split condition can be expressed as $\operatorname{Ext}^{1}\left(\pi^{*} E_{2} \otimes \mathcal{O}_{X}(D), \pi^{*} E_{1} \otimes \mathcal{O}_{X}(-D)\right)=$ $H^{1}\left(X, \mathcal{E} \otimes \mathcal{O}_{X}(-2 D)\right) \neq 0$ where $\mathcal{E}=E_{1} \otimes E_{2}^{*}$. As in [1] applying the Leray spectral 
sequence to $\pi: X \rightarrow B$ yields as sufficient condition for $H^{1}\left(X, \mathcal{E} \otimes \mathcal{O}_{X}(-2 D)\right) \neq 0$ the following condition (for $x<0$ )

$$
\chi\left(B, \mathcal{E} \otimes \mathcal{O}_{B}(-2 \alpha)\right)=4+8 \alpha^{2}-4 \alpha c_{1}-2\left(k_{1}+k_{2}\right)<0
$$

Finally, it remains to determine the range in the Kähler cone where $V_{4}$ is stable, i.e., that for any coherent subsheaf $F$ of rank $0<r<4$ we have $\mu(F)<0$. Solving as in [1] the corresponding inequalities we find for the general Kähler class $J=z \Sigma+h \pi^{*} c_{1}$ where $z, h \in \mathbf{R}$ with $0<z<h$ the range (for $x<0$; here $\zeta:=h-z$ )

$$
\frac{-x c_{1}^{2}}{\left(\alpha-x c_{1}\right) c_{1}} h^{2}<h^{2}-\zeta^{2}<\frac{-x c_{1}^{2}}{\left(\alpha-x c_{1}\right) c_{1}-1} h^{2}
$$

In summary, we find $V_{4}$ is stable if (0.10) and (0.11) are satisfied (the latter just fixes an appropriate range of $z$ ).

Let us now derive sufficient conditions for $H^{1}\left(X, V_{4}\right) \neq 0$ and $H^{1}\left(X, V_{4}^{*}\right) \neq 0$. The Leray spectral sequence gives the following exact sequence

$$
0 \rightarrow H^{1}\left(B, \pi_{*} V_{4}^{*}\right) \rightarrow H^{1}\left(X, V_{4}^{*}\right) \rightarrow
$$

Thus it suffices to show that $H^{1}\left(B, \pi_{*} V_{4}^{*}\right) \neq 0$. For this we apply $\pi_{*}$ to the defining exact sequence of $V_{4}^{*}$ and find the exact sequence

$$
0 \rightarrow E_{2}^{*} \otimes \mathcal{O}_{B}(-\alpha) \otimes \pi_{*} \mathcal{O}_{X}(-x \Sigma) \rightarrow \pi_{*} V_{4}^{*} \rightarrow E_{1}^{*} \otimes \mathcal{O}_{B}(\alpha) \otimes \pi_{*} \mathcal{O}_{X}(x \Sigma) \rightarrow
$$

For $x<0$ one has $\pi_{*} \mathcal{O}_{X}(x \Sigma)=0$ and finds $E_{2}^{*} \otimes \mathcal{O}_{B}(-\alpha) \otimes \pi_{*} \mathcal{O}_{X}(-x \Sigma) \cong \pi_{*} V_{4}^{*}$ It follows

$$
H^{1}\left(B, \pi_{*} V_{4}^{*}\right)=H^{1}\left(B, E_{2}^{*} \otimes \mathcal{O}_{B}(-\alpha) \otimes \pi_{*} \mathcal{O}_{X}(-x \Sigma)\right)
$$

As $\pi_{*} \mathcal{O}_{X}(-x \Sigma)=\mathcal{O}_{B} \oplus \ldots$ it will be sufficient to show that $H^{1}\left(B, E_{2}^{*} \otimes \mathcal{O}_{B}(-\alpha)\right) \neq 0$. The index theorem gives $\chi\left(B, E_{2}^{*} \otimes \mathcal{O}_{B}(-\alpha)\right)=2+\alpha^{2}-\alpha c_{1}-k_{2}$ from which we conclude that

$$
2+\alpha^{2}-\alpha c_{1}-k_{2}<0 \Longrightarrow H^{1}\left(B, E_{2}^{*} \otimes \mathcal{O}_{B}(-\alpha)\right) \neq 0 \Longrightarrow H^{1}\left(X, V^{*}\right) \neq 0
$$

The same reasoning applied for $H^{1}(X, V)$ yields

$$
2+\alpha^{2}-\alpha c_{1}-k_{1}<0 \Longrightarrow H^{1}\left(B, E_{1}^{*} \otimes \mathcal{O}_{B}(-\alpha)\right) \neq 0 \Longrightarrow H^{1}(X, V) \neq 0
$$


Let us now determine the physical constraints we have to impose on $V_{5}$ in order to get a viable standard model compactification of the heterotic string. What concerns the invariance of the deformed bundle one can, as in [1] app. B, argue for the existence of invariant elements in the two non-trivial extension spaces (to solve both conditions simultaneously one can use reflection twists $v \rightarrow-v$ in both fiber vector spaces of $V_{4}$ and $\mathcal{O}_{X}$ ). Further one has still to make sure that the deformability to first order, which we have checked, extends to a full global construction, which we assume can be done.

Note further that the characteristic classes of $V^{\prime}$ are invariant under deformations. Therefore we have $c\left(V_{5}\right)=c\left(V^{\prime}\right)$ and a direct computation yields

$$
\begin{aligned}
& c_{2}\left(V_{5}\right)=-2 x\left(2 \alpha-x c_{1}\right) \Sigma-2 \alpha^{2}+k_{1}+k_{2} \\
& \frac{c_{3}\left(V_{5}\right)}{2}=2 x\left(k_{1}-k_{2}\right)
\end{aligned}
$$

Further one has to satisfy the heterotic anomaly condition $c_{2}(X)-c_{2}\left(V_{5}\right)=[W]=$ $w_{B} \Sigma+a_{f} F$ where $W$ is a space-time filling fivebrane wrapping a holomorphic curve of $X$. This leads to the condition that $[W]$ is an effective curve class in $X$, which in turn can be expressed by the two conditions $w_{B} \geq 0$ and $a_{f} \geq 0$. Inserting the expressions for $c_{2}(X)=6 c_{1} \Sigma+5 c_{1}^{2}+c_{2}$ and $c_{2}(V)$ gives the conditions

$$
\begin{aligned}
& w_{B}=\left(6-2 x^{2}\right) c_{1}+4 x \alpha \geq 0 \\
& a_{f}=44+2 \alpha^{2}-k_{1}-k_{2} \geq 0
\end{aligned}
$$

Finally, the physical net-generation number of chiral fermions, downstairs on $X / \mathbf{Z}_{2}$, is given by

$$
N_{\text {gen }}^{\text {phys }}=x\left(k_{1}-k_{2}\right)
$$

To summarize, we get the following list of constraints (besides $x<0$ )

$$
\begin{aligned}
\alpha c_{1} & >0 \\
2+\alpha^{2}-\alpha c_{1}-k_{i} & <0, \quad \text { where } i=1,2 \\
2+4 \alpha^{2}-2 \alpha c_{1}-\left(k_{1}+k_{2}\right) & <0 \\
\left(6-2 x^{2}\right) c_{1}+4 x \alpha & \geq 0 \\
44+2 \alpha^{2}-\left(k_{1}+k_{2}\right) & \geq 0 \\
x\left(k_{1}-k_{2}\right) & = \pm 3
\end{aligned}
$$


(and $k_{i} \geq 8$ for $h=\frac{1}{2}$, cf. [1], app. B). One realizes that (0.24) entails $x=-1$ and so

$$
\alpha \leq c_{1}
$$

One finds that the following $\alpha$ 's are possible (the entries in $(p, q)$ refer to the multiples of the two generators in $\left.B=\mathbf{P}^{1} \times \mathbf{P}^{1}\right)$ :

$$
\alpha=(-1,2),(1,1),(1,0),(0,2),(1,2),(2,2)
$$

besides interchanging the entries. For instance, one finds then for $\alpha=(1,1)$ that $k_{1}=8+i, k_{2}=11+i$ where $i=0, \ldots, 14$ or $\alpha=(1,0)$ and $i=0, \ldots, 12$ (besides interchanging the $k_{i}$ ).

\section{References}

[1] B. Andreas and G. Curio, Extension bundles and the standard model, hep-th/0703210.

[2] R. Donagi, B. Ovrut, T. Pantev and D. Waldram, Standard-model bundles on non-simply connected Calabi-Yau threefolds, hep-th/0008008, JEHP 0108 (2001) 053.

[3] V. Buchard and R. Donagi, An SU(5) heterotic standard model, hep-th/0512149, Phys. Lett. B633 (2006) 783.

[4] V. Bouchard, M. Cvetic and R. Donagi, Tri-linear couplings in an heterotic minimal supersymmetric standard model, hep-th/0602096, Nucl. Phys. B745 (2006) 62.

[5] R. Blumenhagen, G. Honecker and T. Weigand, Loop-corrected compactifications of the heterotic string with line bundles, hep-th/0504232, JHEP 0506 (2005) 020.

[6] K. Uhlenbeck and S.-T. Yau, On the existence of Hermitian Yang-Mills connections in stable vector bundles, Comm. Pure Appl. Math. 39 (1986), pp. 257-293., Frontiers of the mathematical sciences: 1985 (New York, 1985).

[7] S. Donaldson, Proc. London Math. Soc. 3, 1 (1985).

[8] B. Andreas and G. Curio, Stable bundle extensions on elliptic Calabi-Yau threefolds, math.ag/0611762.

[9] B. Andreas and G. Curio, Heterotic models without fivebranes, hep-th/0611309.

[10] D. Huybrechts, The tangent bundle of a Calabi-Yau manifold - Deformations and restrictions to rational curves, Comm. Math. Phys. 171 (1995), 139.

[11] R. Donagi, R. Reinbacher and S.T. Yau, Yukawa Couplings on Quintic Threefolds, heptth/0605203.

[12] E. Witten, New Issues in Manifolds of SU(3) Holonomy," Nucl. Phys. B 268, 79 (1986).

[13] L. Witten and E. Witten, Large Radius Expansion of Superstring Compactifications, Nucl. Phys. B 281, 109 (1987). 